\title{
Tempo de latência para a leitura: influência da frequiência da palavra escrita e da escolarização
}

\author{
Latency time for reading: influence of frequency of written words and \\ schooling
}

\author{
Luciene Stivanin $^{1}$, Claudia Ines Scheuer ${ }^{2}$
}

\begin{abstract}
RESUMO
Objetivo: Verificar a influência da freqüência das palavras escritas e da escolarização para o tempo de latência. Métodos: Participaram 74 crianças, de $2^{\mathrm{a}}$ a $4^{\mathrm{a}}$ séries de escola pública $(\mathrm{m}=9,6 \mathrm{a})$, ambos os sexos, sem alterações no desenvolvimento e sem queixas escolares. Em computador, com programa particularmente elaborado para esse estudo, aplicou-se um teste de leitura em voz alta de palavras isoladas, caracterizadas por alta e baixa frequiência, variando em regularidade, extensão e complexidade silábica. Resultados: Os dados mostraram que o tempo de latência foi menor para a leitura de palavras freqüentes e houve interação entre série e tipo de palavra. A correlação negativa entre tempo de latência e acertos foi significante, apenas para palavras de baixa frequiência, na $3^{\mathrm{a}} \mathrm{e} 4^{\mathrm{a}}$ séries. Conclusão: A freqüência influenciou diferentemente o tempo de latência e a precisão da leitura, quando levadas em consideração as características das palavras e a escolarização.
\end{abstract}

Descritores: Criança; Leitura; Tempo de reação; Software

\section{INTRODUÇÃO}

A leitura envolve a conversão de uma representação visual em uma representação fonológica ${ }^{(1-3)}$. A leitura requer compreensão da linguagem oral e da estrutura da língua, que pode se dar desde o texto até um único fonema/ grafema, este com e sem sentido próprio. Variáveis lingüísticas relacionadas à palavra interferem no desenvolvimento da leitura, bem como interagem com alterações neste desenvolvimento e nas dificuldades resultantes. Estas variáveis diferem de uma língua para outra, tanto na oralização como na escrita.

Entre as variáveis mais citadas e pesquisadas está a frequiência ${ }^{(4-6)}$, considerada como o número de vezes em que as palavras escritas, pertencentes ao vocabulário de uma lín-

(1) Pós-graduanda do Programa de Pós-Graduação em Ciências da Reabilitação do Departamento de Fisioterapia, Fonoaudiologia e Terapia Ocupacional da Faculdade de Medicina da Universidade de São Paulo - USP - São Paulo (SP), Brasil.

(2) Doutora, Docente do Programa de Pós-Graduação em Ciências da Reabilitação do Departamento de Fisioterapia, Fonoaudiologia e Terapia Ocupacional da Faculdade de Medicina da Universidade de São Paulo - USP - São Paulo (SP), Brasil.

Trabalho realizado no Departamento de Fisioterapia, Fonoaudiologia e Terapia Ocupacional da Universidade de São Paulo - USP - São Paulo (SP), Brasil, com financiamento pela Fundação de Amparo à Pesquisa do Estado de São Paulo (processo 05/60488-0) em forma de bolsa de doutorado.

Endereço para correspondência: Luciene Stivanin. R. Teodoro Sampaio, 632/52, Pinheiros, São Paulo - SP, CEP 05406-000. E-mail: stivanin@usp.br Recebido em: 19/4/2007; Aceito em: 30/8/2007 gua, ocorrem dentro de faixas etárias específicas. De acordo com este número de ocorrências, existirão, então, palavras de alta e de baixa freqüência que se diferenciam ao longo das diferentes faixas etárias, línguas, culturas e escolarização.

A influência da freqüência na leitura pode ser explicada por meio do modelo de dupla-rota, um modelo cognitivo de processamento da informação ${ }^{(5-7)}$, o qual assume que a leitura é realizada por meio de duas rotas: uma semântico-lexical e outra fonológica. A rota semântico-lexical é usada para a leitura de uma palavra previamente armazenada no léxico, acessa e recupera de forma direta o significado da palavra e suas formas fonológicas. Por sua vez, a leitura realizada pela rota fonológica ou sublexical envolve a conversão grafemafonema, para uma palavra não estocada na memória. Este processo ocorre de forma serial, traduzindo grafemas ou grupos de grafemas em fonemas, por meio da aplicação de regras.

Desta forma, as palavras que ocorrem com maior frequiência durante o aprendizado de leitura são lidas mais rapidamente do que as palavras com menor freqüência, por já possuírem representações no léxico. Por outro lado, uma palavra pouco freqüente dispõe de um limiar de ativação mais elevado no léxico do que uma palavra freqüente. Espera-se, portanto, que uma palavra pouco freqüente leve mais tempo para ser ativada e que seja objeto de confusões com outras palavras visualmente semelhantes ${ }^{(8-10)}$.

No entanto, qualquer palavra apesar de sua freqüência se constitui de outras propriedades físicas. No caso de palavras 
escritas, as propriedades visuais dos grafemas associadas às propriedades auditivas dos fonemas determinam a regularidade, complexidade articulatória e a extensão de cada palavra.

Deste modo, a extensão refere-se ao número de grafemas que compõem uma palavra; a complexidade silábica referese à estrutura da palavra, ou seja, à disposição dos grafemas nas sílabas. O número de grafemas e sua organização em sílabas associada à complexidade articulatória produzem as diferenças visuais e orais entre uma palavra e outra ${ }^{(5)}$.

Por sua vez, a regularidade para a leitura diz respeito às regras de conversão grafema-fonema durante a leitura, caracterizando palavras regulares e irregulares. São palavras regulares aquelas que apresentam relação unívoca entre grafema e fonema, e que podem ser independentes ou dependentes do contexto. Por outro lado, palavras irregulares são aquelas cujas relações grafofonêmicas são ambíguas ${ }^{(11-13)}$.

A interação destas variáveis associada à idade e ao tempo de experiência com a leitura é um fator que contribui para aumentar as representações das palavras no léxico, acarretando o uso cada vez menor da rota fonológica e permitindo uma leitura mais rápida e precisa ${ }^{(14)}$. Rapidez e precisão na leitura de palavras implicam identificação de uma palavra anteriormente conhecida e em uma nova, não pertencente ao léxico.

Uma das formas de verificar rapidez e precisão no acesso à palavra escrita é por meio do tempo de latência. Este tempo de acesso ao léxico para a leitura de uma palavra depende, dentre outros fatores, das características da língua e é considerado o tempo entre a apresentação do estímulo e o início da resposta ${ }^{(15)} \mathrm{e}$, neste estudo, trata-se da leitura de palavras escritas. É uma medida que tem sido utilizada em estudos sobre os processos envolvidos na compreensão da linguagem ${ }^{(16-17)}$, na leitura, no reconhecimento de palavras escritas $^{(18-20)}$ e na nomeação de figuras ${ }^{(21-26)}$.

O objetivo desta pesquisa foi verificar a influência da freqüência da palavra escrita para o tempo de latência e para a precisão da leitura, levando-se em conta as características da palavra (regularidade, extensão e complexidade silábica) e a escolaridade $\left(2^{\mathrm{a}}, 3^{\mathrm{a}}\right.$ e $4^{\mathrm{a}}$ séries $)$.

\section{MÉTODOS}

Esta pesquisa foi aprovada pela Comissão de Ética para Análise de Projetos de Pesquisa do Hospital das Clínicas da Faculdade de Medicina da Universidade de São Paulo (protocolo de pesquisa $n^{\circ} 637 / 03$ ). Os responsáveis pelas crianças assinaram o Termo de Consentimento Pós-Informação do Hospital das Clínicas da Faculdade de Medicina da Universidade de São Paulo.

\section{Participantes}

Participaram deste estudo 74 crianças leitoras, de $2^{\mathrm{a}}, 3^{\mathrm{a}} \mathrm{e}$ $4^{\mathrm{a}}$ séries do Ensino Fundamental da escola pública municipal Professor Olavo Pezzotti, localizada em São Paulo (SP), cujas famílias apresentaram autorização do responsável legal. A idade cronológica foi de sete a 12 anos, ambos os sexos e consideradas boas falantes, sem alterações no desenvolvimento da linguagem, histórico de fracasso escolar, repetência, evasão e indicação de classe especial, déficits visuais não corrigidos e queixas de problemas auditivos informados por pais e professores.

Foram excluídas as crianças que, durante a aplicação das provas, apresentassem alterações fonológicas e/ou articulatórias, não identificadas por pais e professores. Também foram excluídas aquelas que apresentaram dificuldades na compreensão do teste e/ou que não conseguiram realizar todas as provas.

A distribuição dos sujeitos quanto à idade e gênero pode ser observada nas Tabelas 1 e 2, respectivamente.

Tabela 1. Distribuição dos sujeitos por idade (em meses)

\begin{tabular}{lcccc}
\hline & \multicolumn{4}{c}{ Séries } \\
& $2^{\mathrm{a}}$ & $3^{\mathrm{a}}$ & $4^{\mathrm{a}}$ & Total \\
\hline Média & 103,28 & 114,58 & 128,64 & 115,51 \\
Desvio padrão & 3,96 & 5,78 & 7,89 & 12,11 \\
$\mathrm{n}$ & 25 & 24 & 25 & 74 \\
\hline
\end{tabular}

Para conhecer o perfil sócio econômico das famílias das crianças deste estudo utilizou-se a escala ABIPEME(27), cujos resultados estão na Tabela 3 .

\section{Materiais e procedimentos}

Para a seleção/caracterização dos sujeitos deste estudo foram utilizados: Termo de Consentimento Livre e Esclarecido, Anamnese, Questionário Escolar e Escala de Avaliação do Nível Sócio-Econômico Familiar da Associação Brasileira de Institutos de Pesquisa de Mercado - ABIPEME ${ }^{(27)}$.

Para a verificação da influência da frequiência foi aplicada uma prova de leitura em voz alta de palavras isoladas, contendo palavras de alta e de baixa freqüência selecionadas do tra-

Tabela 2. Distribuição dos sujeitos por gênero

\begin{tabular}{|c|c|c|c|c|c|c|c|c|}
\hline \multirow[b]{3}{*}{ Gênero } & \multicolumn{6}{|c|}{ Séries } & & \\
\hline & \multicolumn{2}{|c|}{$2^{a}$} & \multicolumn{2}{|c|}{$3^{a}$} & \multicolumn{2}{|c|}{$4^{a}$} & \multicolumn{2}{|c|}{ total } \\
\hline & $\mathrm{n}$ & $\%$ & $\mathrm{n}$ & $\%$ & $\mathrm{n}$ & $\%$ & $\mathrm{n}$ & $\%$ \\
\hline Feminino & 13 & $52,0 \%$ & 12 & $50,0 \%$ & 14 & $56,0 \%$ & 39 & $52,7 \%$ \\
\hline Masculino & 12 & $48,0 \%$ & 12 & $50,0 \%$ & 11 & $44,0 \%$ & 35 & $47,3 \%$ \\
\hline Total & 25 & $100,0 \%$ & 24 & $100,0 \%$ & 25 & $100,0 \%$ & 74 & $100,0 \%$ \\
\hline
\end{tabular}


Tabela 3. Distribuição dos sujeitos por classe sócio-econômico-familiar

\begin{tabular}{|c|c|c|c|c|c|c|c|c|}
\hline \multirow[b]{3}{*}{ ABIPEME } & \multicolumn{6}{|c|}{ Séries } & \multirow{2}{*}{\multicolumn{2}{|c|}{ total }} \\
\hline & \multicolumn{2}{|c|}{$2^{a}$} & \multicolumn{2}{|c|}{$3^{a}$} & \multicolumn{2}{|c|}{$4^{a}$} & & \\
\hline & $\mathrm{n}$ & $\%$ & $\mathrm{n}$ & $\%$ & $\mathrm{n}$ & $\%$ & $\mathrm{n}$ & $\%$ \\
\hline A & 0 & 0 & 0 & 0 & 1 & 4,0 & 1 & 1,4 \\
\hline B & 10 & 40,0 & 7 & 29,2 & 6 & 24,0 & 23 & 31,1 \\
\hline C & 13 & 52,0 & 12 & 50,0 & 13 & 52,0 & 38 & 51,4 \\
\hline D & 2 & 8,0 & 5 & 20,8 & 2 & 8,0 & 9 & 12,2 \\
\hline E & 0 & 0 & 0 & 0 & 3 & 12,0 & 3 & 4,1 \\
\hline Total & 25 & 100,0 & 24 & 100,0 & 25 & 100,0 & 74 & 100,0 \\
\hline
\end{tabular}

Legenda: A: classe alta; B: classe média; C: classe média baixa; D: classe baixa; E: classe muito baixa

balho de Pinheiro ${ }^{(28)}$, com crianças na faixa pré-escolar e séries iniciais. As palavras de baixa freqüência são caracterizadas por ocorrência de uma a 30 vezes no material escrito enquanto as de alta frequiência, por ocorrência de 73 a 14064.

Para este trabalho, as palavras de alta frequiência referem-se às mais escritas para crianças de $2^{\mathrm{a}}$ série do Ensino Fundamental. As palavras de baixa freqüência, ou as menos ocorrentes ou escritas, foram escolhidas aquelas apareceram repetidamente para as $2^{\mathrm{a}}, 3^{\mathrm{a}}$ e $4^{\mathrm{a}}$ séries.

As palavras de alta e de baixa freqüência foram caracterizadas pela complexidade silábica, extensão e regularidade. Quanto à extensão, foram considerados quatro e seis grafemas para palavras de estrutura simples, e cinco ou mais grafemas para as palavras com estrutura complexa.

Quanto à complexidade silábica, os nomes foram caracterizados por estrutura silábica CV (consoante-vogal); $\mathrm{CCV}$ (consoante-consoante-vogal) e CVC (consoante-vogal-consoante). Não foram incluídos os dígrafos. As palavras também foram selecionadas por sua regularidade e irregularidade para a leitura.

De acordo com o balanceamento descrito, foram formados 12 grupos de palavras escritas, em um total de 96 palavras:

Grupo 1 - alta freqüência, quatro grafemas, CVCV, regular: casa, galo, sapo, boné, pato, rato, vaca, vaso.

Grupo 2 - alta freqüência, quatro grafemas, $C V C V$, irregular: bola, mesa, bolo, lixo, boca, rosa, dedo.

Grupo 3 - alta freqüência, seis grafemas, CVCVCV, regular: cavalo, girafa, sapato, macaco, jacaré, menino, menina, mágico.

Grupo 4 - alta freqüência, seis grafemas, CVCVCV, irregular: boneca, janela, raposa, cabeça.

Grupo 5 - alta freqüência, extensão variável, outras estruturas, regular: livro, prato, braço, lápis, nuvem, gravata, ônibus, árvore, vestido, elefante, laranja.

Grupo 6 - alta freqüência, extensão variável, outras estruturas, irregular: cobra, porta, corpo, igreja, estrela, caderno.

Grupo 7 - baixa freqüência, quatro grafemas, CVCV, regular: sofá, faca, laço, maçã, fita, jipe, taça.

Grupo 8 - baixa freqüência, quatro grafemas, CVCV, irregular: bola, seta, pote, foca, táxi, selo, lobo.

Grupo 9 - baixa freqüência, seis grafemas, CVCVCV, regu- lar: banana, barata, batata, tomate, cabide, camisa, coruja, cálice, casaco, pirata.

Grupo 10 - baixa freqüência, seis grafemas, CVCVCV, irregular: bexiga, tigela, xícara, cabelo, caneta, cebola, cereja, colete, panela, gaveta.

Grupo 11 - baixa freqüência, extensão variável, outras estruturas, regular: grilo, metrô, tigre, tampa, cabrito, óculos, escada, lagarto, morango, melancia.

Grupo 12 - baixa freqüência, extensão variável, outras estruturas, irregular: cofre, prego, zebra, porco, torta, frigideira, escova, martelo.

As palavras escritas foram impressas em negrito, fonte Microsoft Sans Serif e tamanho 40, localizadas no centro da tela (fundo branco) de um computador e apresentadas isoladamente. A criança foi instruída a ler a palavra escrita em voz alta assim que esta aparecesse na tela.

Para a obtenção do tempo de latência, foi desenvolvido um programa especialmente para esta pesquisa, elaborado no Departamento de Engenharia da Computação, na Universidade de Campinas (SP). O sistema utilizado para a criação do programa foi o JAVA e instalado em computador portátil com as configurações: HP Pavilion, modelo ze5200, processador Pentium 4, $2.4 \mathrm{GHz}, 512$ de memória, $40 \mathrm{~Gb}$, CDRW, matriz ativa 15 ", rede 10/100, sistema operacional Windows XP Home; microfone Bright.

Durante a aplicação das provas, o programa é capaz de capturar os sinais emitidos pelo microfone acoplado ao computador e as bibliotecas de som convertem os sinais elétricos em valores medidos com precisão de oito casas. Estes valores são tratados e a curva referente à leitura de cada palavra e de cada criança é desenhada. Manualmente, o pesquisador localiza e clica no início da vocalização da criança, após visualizar a onda e ouvir a produção oral determinando assim o tempo de latência. Os dados obtidos na análise ficam armazenados em arquivos individuais e podem ser acessados no Programa Excel conectado ao programa computacional.

A leitura realizada de forma ortográfica ${ }^{(29)}$ recebeu um (1) ponto por ser a mais precisa. Outras formas de leitura, menos precisas, foram classificadas de acordo com os critérios propostos por outros autores ${ }^{(30)}$ :

1- Neologismo: a resposta da criança gera uma não-palavra ou uma pseudopalavra diferente do estímulo alvo (ex.: cadeira lido como daqueira). 
2- Regularização: a pronúncia grafema-fonema irregular de uma palavra é substituída por uma pronúncia regular e mais fraca (boxe lido como boche).

3- Desconhecimento da regra ortográfica: pronúncias incorretas devido ao desconhecimento de certas regras contextuais da língua (casa lida como cassa).

4- Desconhecimento da regra de acentuação: a pronúncia da palavra não leva em conta as regras de acentuação (parágrafo lido como paragráfo).

5- Paralexia verbal formal: substituição lexical mantendo uma semelhança de estrutura de letras, sem relação semântica (tomou lido como tomate).

6- Lexicalização: uma pseudopalavra é lida como uma palavra real, mantendo relações de semelhança formal (azercico lido como exercício).

7- Não resposta: quando não foi enunciada nenhuma pronúncia ao estímulo apresentado.

A leitura realizada com substituições, mas com segmentações, repetições e/ou prolongamentos, foi classificada na categoria erros de produção. Quando a criança leu a palavra inadequadamente, mas logo em seguida a corrigiu, a classificação foi denominada revisão.

\section{Análise}

Foram computadas a média do tempo de latência e a porcentagem de acertos para a leitura em cada grupo de palavras e em cada série. Para a comparação entre palavras de alta e de baixa frequiência, e entre as séries, foi utilizado o teste estatístico ANOVA. Para a correlação entre o tempo de latência e os acertos, foi empregado o teste de correlação de Pearson.

\section{RESULTADOS}

A seguir, estão descritos os resultados referentes à leitura de palavras. A média do tempo de latência e o percentual de acertos de leitura podem ser visualizados nas Tabelas 4 e 6 ,

Tabela 4. Média e desvio-padrão do tempo de latência para a leitura de palavras em cada série e tipo de palavra

\begin{tabular}{|c|c|c|c|}
\hline \multirow{3}{*}{ Tipos de palavras } & \multicolumn{3}{|c|}{ séries } \\
\hline & $2^{a}$ & $3^{a}$ & $4^{\mathrm{a}}$ \\
\hline & M (DP) & $\mathrm{M}(\mathrm{DP})$ & $\mathrm{M}(\mathrm{DP})$ \\
\hline $\mathrm{AF}, 4 \mathrm{G}, \mathrm{CV}, \mathrm{R}$ & $1,20(0,35)$ & $1,06(0,46)$ & $1,10(0,45)$ \\
\hline $\mathrm{AF}, 4 \mathrm{G}, \mathrm{CV}, \mathrm{I}$ & $1,08(0,25)$ & $1,08(0,46)$ & $0,99(0,28)$ \\
\hline$A F, 6 G, C V, R$ & $1,22(0,41)$ & $1,20(0,58)$ & $1,15(0,43)$ \\
\hline $\mathrm{AF}, 6 \mathrm{G}, \mathrm{CV}, \mathrm{I}$ & $1,28(0,44)$ & $1,22(0,54)$ & $1,29(0,60)$ \\
\hline $\mathrm{AF}, 5 \mathrm{e}+\mathrm{G}, \mathrm{OE}, \mathrm{R}$ & $1,27(0,37)$ & $1,14(0,41)$ & $1,16(, 45)$ \\
\hline $\mathrm{AF}, 5$ e +G, OE, I & $1,44(0,74)$ & $1,26(0,57)$ & $1,30(0,64)$ \\
\hline $\mathrm{BF}, 4 \mathrm{G}, \mathrm{CV}, \mathrm{R}$ & $1,09(0,25)$ & $1,24(0,61)$ & $1,17(0,51)$ \\
\hline $\mathrm{BF}, 4 \mathrm{G}, \mathrm{CV}, \mathrm{I}$ & $1,28(0,42)$ & $1,26(0,58)$ & $1,19(0,55)$ \\
\hline $\mathrm{BF}, 6 \mathrm{G}, \mathrm{CV}$ & $1,41(0,56)$ & $1,33(0,64)$ & $1,34(0,57)$ \\
\hline $\mathrm{BF}, 6 \mathrm{G}, \mathrm{CV}$ & $1,50(0,62)$ & $1,39(0,65)$ & $1,31(0,58)$ \\
\hline $\mathrm{BF}, 5 \mathrm{e}+\mathrm{G}, \mathrm{OE}, \mathrm{R}$ & $1,35(0,50)$ & $1,30(0,52)$ & $1,31(0,61)$ \\
\hline $\mathrm{BF}, 5$ e +G, OE, I & $1,41(0,52)$ & $1,46(0,82)$ & $1,32(0,58)$ \\
\hline
\end{tabular}

Legenda: M- média; $\mathrm{DP}$ - desvio-padrão; $\mathrm{AF}$ : alta freqüência; $\mathrm{BF}$ : baixa freqüência; G: grafemas;CV:consoante-vogal; OE: outras estruturas; R: regular; I:irregular respectivamente. Os p-valores da comparação entre os tipos de palavras e entre as séries são apresentados n (tempo de latência) e 7 (acertos). Na Tabela 8, podem ser observadas as características da leitura e na Tabela 9, a correlação entre tempo de latência e acertos.

Tabela 5. P-valores da comparação entre palavras de alta e baixa freqüência para o tempo de latência na prova de leitura

\begin{tabular}{|c|c|c|c|}
\hline \multirow{2}{*}{$\begin{array}{l}\text { Alta x baixa } \\
\text { freqüência }\end{array}$} & \multicolumn{3}{|c|}{ p-valores } \\
\hline & Série & Palavra & Série $\mathrm{x}$ palavra \\
\hline $4 G, C V, R$ & 0,9915 & 0,1398 & 0,0014 * \\
\hline $4 G, C V, I$ & 0,6909 & $<0,0001$ * & 0,9666 \\
\hline $6 \mathrm{G}, \mathrm{CV}, \mathrm{R}$ & 0,8602 & $<0,0001$ * & 0,7806 \\
\hline $6 \mathrm{G}, \mathrm{CV}, \mathrm{I}$ & 0,8009 & 0,0018 * & 0,1672 \\
\hline $5 e+\mathrm{G}, \mathrm{OE}, \mathrm{R}$ & 0,7694 & $<0,0001$ * & 0,3723 \\
\hline 5 e +G, OE, I & 0,8051 & 0,2084 & 0,1080 \\
\hline
\end{tabular}

$\mathrm{p}<0,05^{*}$

Legenda: G: grafemas, CV: consoante-vogal; R:regular; I: irregular;OE: outras estruturas

Tabela 6. Percentual de acertos para a leitura de palavras em cada série e tipo de palavra

\begin{tabular}{|c|c|c|c|}
\hline \multirow{3}{*}{ Tipos de palavras } & \multicolumn{3}{|c|}{ Séries } \\
\hline & $2^{\mathrm{a}}$ & $3^{a}$ & $4^{a}$ \\
\hline & $\mathrm{M}(\mathrm{DP})$ & $\mathrm{M}(\mathrm{DP})$ & $\mathrm{M}(\mathrm{DP})$ \\
\hline $\mathrm{AF}, 4 \mathrm{G}, \mathrm{CV}, \mathrm{R}$ & $93,0(9,6)$ & $100,0(0,0)$ & $97,5(5,1)$ \\
\hline $\mathrm{AF}, 4 \mathrm{G}, \mathrm{CV}, \mathrm{I}$ & $96,0(6,5)$ & $95,2(8,1)$ & $96,0(7,7)$ \\
\hline AF, 6G, CV, R & $97,0(5,4)$ & $99,0(3,5)$ & $97,0(6,5)$ \\
\hline AF, 6G, CV, I & $97,0(8,3)$ & $97,9(7,1)$ & $97,0(8,3)$ \\
\hline $\mathrm{AF}, 5 \mathrm{e}+\mathrm{G}, \mathrm{OE}, \mathrm{R}$ & $96,7(6,4)$ & $98,5(35)$ & $97,8(6,0)$ \\
\hline $\mathrm{AF}, 5 \mathrm{e}+\mathrm{G}, \mathrm{OE}, \mathrm{I}$ & $96,0(11,1)$ & $97,2(8,0)$ & $94,0(14,3)$ \\
\hline $\mathrm{BF}, 4 \mathrm{G}, \mathrm{CV}, \mathrm{R}$ & $95,4(9,9)$ & $98,2(4,8)$ & $95,4(9,9)$ \\
\hline $\mathrm{BF}, 4 \mathrm{G}, \mathrm{CV}, \mathrm{I}$ & $85,1(16,2)$ & $90,5(11,7)$ & $86,9(15,4)$ \\
\hline $\mathrm{BF}, 6 \mathrm{G}, \mathrm{CV}, \mathrm{R}$ & $90,8(6,4)$ & $83,3(8,2)$ & $94,8(5,1)$ \\
\hline $\mathrm{BF}, 6 \mathrm{G}, \mathrm{CV}, \mathrm{I}$ & $89,6(12,4)$ & $80,8(16,7)$ & $93,6(10,8)$ \\
\hline $\mathrm{BF}, 5 \mathrm{e}+\mathrm{G}, \mathrm{OE}, \mathrm{R}$ & $91,2(11,3)$ & $80,4(10,0)$ & $93,2(6,9)$ \\
\hline $\mathrm{BF}, 5 \mathrm{e}+\mathrm{G}, \mathrm{OE}, \mathrm{I}$ & $94,0(7,3)$ & $94,3(9,7)$ & $94,0(8,9)$ \\
\hline
\end{tabular}

Legenda: M-média; DP- desvio-padrão; $\mathrm{AF}$ : alta freqüência; $\mathrm{BF}$ : baixa freqüência; G: grafemas;CV:consoante-vogal; OE: outras estruturas; R: regular; I:irregular

Tabela 7. P-valores da comparação entre palavras de alta e baixa freqüência para acertos na prova de leitura

\begin{tabular}{|c|c|c|c|}
\hline \multirow{2}{*}{$\begin{array}{l}\text { Alta x baixa } \\
\text { freqüência }\end{array}$} & \multicolumn{3}{|c|}{$p$-valores } \\
\hline & Série & Palavra & Série x palavra \\
\hline $4 \mathrm{G}, \mathrm{CV}, \mathrm{R}$ & 0,0206 * & 0,6554 & 0,1585 \\
\hline $4 \mathrm{G}, \mathrm{CV}, \mathrm{I}$ & 0,6941 & $<0,0001$ * & 0,2746 \\
\hline $6 \mathrm{G}, \mathrm{CV}, \mathrm{R}$ & 0,0022 * & $<0,0001$ * & $<0,0001^{*}$ \\
\hline $6 \mathrm{G}, \mathrm{CV}, \mathrm{I}$ & $0,0062^{*}$ & $<0,0001$ * & 0,0021 * \\
\hline 5 e $+\mathrm{G}, \mathrm{OE}, \mathrm{R}$ & $0,0130^{\star}$ & $<0,0001^{\star}$ & $<0,0001^{*}$ \\
\hline 5 e $+\mathrm{G}, \mathrm{OE}, \mathrm{I}$ & 0,7673 & 0,2196 & 0,6552 \\
\hline
\end{tabular}

$\mathrm{p}<0,05^{*}$

Legenda: G: grafemas, CV: consoante-vogal; R:regular; I: irregular;OE: outras estruturas 
Tabela 8. Características da leitura em cada tipo de palavra e em cada série

\begin{tabular}{llll}
\hline Palavras & & séries & $4^{\mathrm{a}}$ \\
\hline AF, 4G, CV, R & $2^{\mathrm{a}}$ & $3^{\mathrm{a}}$ & DRO \\
AF, 4G, CV, I & DRO, DRA, PVF & DRO, PVF, R \\
AF, 6G, CV, R & DRO, PVF, R & DRO R & DRO \\
AF, 6G, CV, I & DRO, PVF, N & DRO, N & DRO, PVF \\
AF, 5 e +G, OE, R & DRO & DRO, DRA, N & DRO \\
AF, 5 e +G, OE, I & DRO, PVF, N, RP & DRO, N & DRO, PVF, N \\
BF, 4G, CV, R & DRO, PVF, NR & DRO, PVF & DRO, PVF \\
BF, 4G, CV, I & DRO, DRA, PVF & DRO, DRA, R & DRO, DRA, R \\
BF, 6G, CV, R & DRO, PVF, R & DRO, DRA & DRO, DRA, PVF \\
BF, 6G, CV, I & DRO, DRA, PVF, & DRO, N & DRO, N \\
BF, 5 e +G, OE, R & DRO, PVF, R, N & DRO,DRA,PVF,N, NR & DR, DRA, N \\
BF, 5 e +G, OE, I & DRO, DRA, PVF, NR & DRO, PVF, R & DRO, PVF, R
\end{tabular}

Legenda: AF: alta freqüência; BF: baixa freqüência; G: grafemas; CV:consoante-vogal; OE: outras estruturas; R: regular; I:irregular; DRO: desconhecimento da regra ortográfica; DRA: desconhecimento da regra de acentuação, PVF: paralexia verbal formal; R: regularização; N: neologismo; NR: não resposta; RP: resposta parcial

De acordo com os dados das Tabelas 4 e 5 , o tempo de latência foi menor para palavras de alta freqüência do que nas de baixa, em todos os tipos de palavras, com exceção das palavras mais simples $(4 \mathrm{G}, \mathrm{CV}$ e $\mathrm{R})$ e das mais complexas $(5 \mathrm{e}+\mathrm{G}, \mathrm{OE}, \mathrm{I})$. Entre as séries, o tempo de latência foi homogêneo. A interação significativa entre série e palavra no grupo de palavras com quatro grafemas, $\mathrm{CV}$ e regulares deve-se ao fato da mudança de comportamento entre as séries com relação às palavras de alta $\mathrm{e}$ baixa freqüência. Isto quer dizer que, enquanto a $3^{\mathrm{a}} \mathrm{e}$ a $4^{\mathrm{a}}$ séries apresentaram tempo de latência menor para palavras de alta frequiência e maior para as de baixa, as crianças de $2^{2}$ série apresentaram o comportamento inverso.

Os dados das Tabelas 6 e 7 apontam para maior percentual de acertos na leitura de palavras de alta do que nas de baixa frequiência. Assim como ocorreu com relação ao tempo de latência, esta diferença entre palavras de alta e de baixa freqüência não foi observada nos tipos de palavras mais simples $(4 \mathrm{G}, \mathrm{CV}, \mathrm{R})$ e mais complexas $(5 \mathrm{e}+\mathrm{G}, \mathrm{OE}, \mathrm{I})$. O comportamento entre as séries foi semelhante.

As características da leitura também foram qualitativamente analisadas. Devido à baixa incidência, não há teste estatístico aplicável.

Observou-se nas características da leitura que DRO (desconhecimento da regra ortográfica), DRA (desconhecimento da regra de acentuação) e R (regularização) foram apresentados em maior quantidade pelas crianças da $2^{\mathrm{a}}$ série, embora não houvesse diferença estatística. As diferenças significantes foram observadas entre as $2^{\mathrm{a}} \mathrm{e} 4^{\mathrm{a}}$ séries (negrito). Considerando-se a concentração de respostas, estas passam de uma concentração em DRA (desconhecimento da regra de acentuação) na $2^{\mathrm{a}}$ série para uma concentração em PVF (paralexia verbal formal) e $\mathrm{N}$ (neologismo) na $4^{\mathrm{a}}$ série.

As crianças da $4^{\text {a }}$ série apresentaram menor quantidade de fragmentações nas respostas (erros de produção), de forma estatisticamente significante. Por outro lado, os alunos de $3^{\mathrm{a}}$ e $4^{\mathrm{a}}$ séries realizaram mais revisões nas suas respostas do que a $2^{a}$ série.

A porcentagem de erros de produção ocorridos durante a leitura adequada das palavras não foi estatisticamente diferente entre as séries $(\mathrm{p}=0,0612)$.

Neste teste estatístico da Tabela 9, a correlação negativa indica que, enquanto o valor de uma variável aumenta, o da

Tabela 9. Correlação entre tempo de latência e acertos em cada prova e série para cada grupo de palavras

\begin{tabular}{|c|c|c|c|c|}
\hline \multirow[t]{2}{*}{ Tipos de palavras } & & \multicolumn{3}{|c|}{ Leitura } \\
\hline & & $2^{a}$ & $3^{\mathrm{a}}$ & $4^{\mathrm{a}}$ \\
\hline \multirow[t]{2}{*}{$A F, 4 G, C V, R$} & C.P. & $-0,399\left(^{*}\right)$ & (a) & $-0,347$ \\
\hline & (p) & 0,048 & - & 0,09 \\
\hline \multirow[t]{2}{*}{$\mathrm{AF}, 4 \mathrm{G}, \mathrm{CV}, \mathrm{I}$} & C.P. & 0,128 & 0,009 &,$- 451\left(^{*}\right)$ \\
\hline & (p) & 0,542 & 0,967 & 0,024 \\
\hline \multirow[t]{2}{*}{$A F, 6 G, C V, R$} & C.P. & $-0,159$ & 0,136 &,$- 417\left(^{*}\right)$ \\
\hline & (p) & 0,447 & 0,525 & 0,038 \\
\hline \multirow[t]{2}{*}{$\mathrm{AF}, 6 \mathrm{G}, \mathrm{CV}, \mathrm{I}$} & C.P. & 0,079 & $-0,267$ & 0,105 \\
\hline & (p) & 0,707 & 0,207 & 0,617 \\
\hline \multirow[t]{2}{*}{$\mathrm{AF}, 5 \mathrm{e}+\mathrm{G}, \mathrm{OE}, \mathrm{R}$} & C.P. & $-0,044$ & 0,023 & $-0,384$ \\
\hline & (p) & 0,836 & 0,917 & 0,058 \\
\hline \multirow[t]{2}{*}{$A F, 5$ e+G, OE, I } & C.P. & $-0,253$ & $-0,115$ & $-0,138$ \\
\hline & (p) & 0,222 & 0,594 & 0,509 \\
\hline \multirow[t]{2}{*}{$\mathrm{BF}, 4 \mathrm{G}, \mathrm{CV}, \mathrm{R}$} & C.P. & $-0,006$ &,$- 407\left(^{*}\right)$ &,$- 427\left(^{\star}\right)$ \\
\hline & (p) & 0,977 & 0,048 & 0,033 \\
\hline \multirow[t]{2}{*}{$\mathrm{BF}, 4 \mathrm{G}, \mathrm{CV}, \mathrm{I}$} & C.P. & 0,1 &,$- 608(* \star)$ &,$- 687\left(^{\star \star}\right)$ \\
\hline & (p) & 0,636 & 0,002 & 0 \\
\hline \multirow[t]{2}{*}{$\mathrm{BF}, 6 \mathrm{G}, \mathrm{CV}, \mathrm{R}$} & C.P. & $-0,022$ &,$- 406\left(^{*}\right)$ & $-0,244$ \\
\hline & (p) & 0,915 & 0,049 & 0,239 \\
\hline \multirow[t]{2}{*}{$\mathrm{BF}, 6 \mathrm{G}, \mathrm{CV}, \mathrm{I}$} & C.P. & 0,117 & $-0,445\left(^{*}\right)$ & $-0,399\left(^{*}\right)$ \\
\hline & (p) & 0,578 & 0,049 & 0,108 \\
\hline \multirow[t]{2}{*}{$\mathrm{BF}, 5 \mathrm{e}+\mathrm{G}, \mathrm{OE}, \mathrm{R}$} & C.P. & $-0,339$ &,$- 605\left(^{\star \star}\right)$ &,$- 397\left(^{*}\right)$ \\
\hline & (p) & 0,097 & 0,002 & 0,05 \\
\hline \multirow[t]{2}{*}{$\mathrm{BF}, 5 \mathrm{e}+\mathrm{G}, \mathrm{OE}, \mathrm{I}$} & C.P. & $-0,165$ & $-0,181$ &,$- 666\left({ }^{\star \star}\right)$ \\
\hline & (p) & 0,43 & 0,397 & 0 \\
\hline
\end{tabular}

Legenda: CP: Correlação de Pearson AF: alta freqüência; $\mathrm{BF}$ : baixa freqüência; $\mathrm{G}$ : grafemas; $\mathrm{CV}$ :consoante-vogal; $\mathrm{OE}$ : outras estruturas; $\mathrm{R}$ : regular; I:irregular * Correlação significante a 0,05.

** Correlação significante a 0,01.

(a) não aplicável, uma das variáveis é constante 
outra diminui. Os dados, então, apontam para correlação negativa estatisticamente significante para todos os grupos de palavras de baixa frequiência, na $3^{\mathrm{a}}$ e $4^{\mathrm{a}}$ séries. Isto indica que o tempo de latência determina a eficiência na leitura de palavras, pior para as de baixa freqüência.

\section{DISCUSSÃO}

Esta pesquisa teve como objetivo principal analisar a influência da frequiência das palavras escritas para o tempo de latência e para a precisão de leitura, ou seja, se a alta ou baixa freqüência determina um menor ou maior tempo de acesso ao léxico, e em qual fase de escolarização.

Considerando-se os tipos de palavras, observou-se que o tempo de latência foi menor para as palavras mais freqüentes do que nas de baixa frequiência, assim como as primeiras foram mais bem lidas do que as últimas, o que está de acordo com a literatura ${ }^{(5,8-10)}$.

A diferença no tempo de latência entre palavras de alta e baixa freqüência foi observada na leitura de quase todos os tipos de palavras (caracterizados por extensão, complexidade silábica e regularidade). Porém, nas palavras mais simples (com quatro grafemas, estrutura silábica $\mathrm{CV}$ e regulares) e nas mais complexas (cinco e mais grafemas, estruturas mais complexas além de CV e irregulares), esta diferença não foi observada.

Desta forma, as três variáveis psicolingüísticas consideradas neste estudo não podem analisadas isoladamente, pois elas interagem causando efeitos no processamento das palavras escritas. Palavras de alta frequiência, regulares e curtas podem ser recuperadas rapidamente do léxico, sem a necessidade da ativação da via fonológica. Porém, as palavras menos frequientes e extensas necessitam da aplicação de regras grafofonêmicas porque não estão estabilizadas na memória; se estas possuírem associações irregulares, ocorre a ativação entre dois fonemas para representar um grafema e a competição entre eles retarda a ativação da forma fonológica correta ${ }^{(5)}$.

Associando o tempo de latência com a escolarização, conforme a criança aumenta a freqüência do contato com a palavra escrita e aprende as regras ortográficas, as representações (fonológicas, ortográficas e de significado) se estabilizam e possibilitam uma recuperação cada vez mais automática e rápida da palavra escrita. Por outro lado, ao ler palavras novas ou pouco freqüentes, extensas e com irregularidades ainda não aprendidas ou em fase de aprendizagem, a criança tem que utilizar regras de conversão entre grafemas e fonemas e/ou realizar uma pesquisa no léxico à procura da representação correspondente ao input, o que lentifica o tempo de recuperação.

Quanto à porcentagem de acertos, ressalta-se que se fosse utilizado apenas o critério acerto/erro, todos os escolares teriam comportamento semelhante. Porém, com a associação deste critério com o tempo de latência e com as estratégias envolvidas na leitura, pôde-se caracterizar o perfil destes escolares durante o processamento da palavra escrita.

Neste sentido, verificou-se que as crianças menos escolarizadas de $2^{\text {a }}$ série utilizam preferencialmente a rota fonológica, o que explica a leitura caracterizada por substitui- ções ortográficas (como "casa" lida como /cassa/), desconhecimento da regra de acentuação ("barata" lida como /baratá/, regularizações ("táxi” lido como /táchi/) e erros de produção (segmentações, repetições, pausas). Este perfil é o mesmo observado em outros estudos sobre o desenvolvimento da leitu$\mathrm{ra}^{(19,25,30)}$.

Com a escolarização e o desenvolvimento, a criança aprende e estabiliza as relações ortográficas, o que permite a recuperação automática e adequada da palavra e conduz ao uso menos freqüiente da rota fonológica. A utilização da rota lexical torna-se mais utilizada e o perfil da leitura muda: as paralexias verbais e os neologismos ${ }^{(30)}$ tornam-se mais freqüentes, além do uso de revisões que se desenvolvem com o amadurecimento do indivíduo. Assim, na tentativa de recuperar a palavra diretamente do léxico, as crianças deixam de regularizá-la e de segmentá-la, incorrendo na seleção de palavras com estrutura fonológica similar ("livro" lido /vidro/ ou "pipoca" lida como /popoca/). As revisões indicam que as crianças mais velhas e escolarizadas são capazes de perceber que a pronúncia realizada não existe e/ou não corresponde à palavra escrita.

No entanto, estas crianças mais velhas e escolarizadas ainda utilizam a rota fonológica na leitura de palavras mais complexas. De acordo com a literatura, a rota lexical está mais desenvolvida a partir da $3^{\mathrm{a}}$ série $^{(30)}$, porém a rota fonológica continua a ser utilizada na leitura de palavras mais complexas juntamente à rota lexical, mesmo na vida adulta, quando o leitor se depara com palavras desconhecidas ou pouco vistas ${ }^{(19)}$.

Sabe-se o reconhecimento da palavra escrita deve ser realizado em tempo suficiente para diminuir a sobrecarga na memória de trabalho e permitir à criança se dedicar aos processos de compreensão da mensagem escrita ${ }^{(31-32)}$. Porém, no presente estudo, considerando a relação do tempo de processamento com a eficiência na leitura, os dados apontaram que recuperar mais rápido não determina uma leitura mais precisa, pois as crianças podem ser mais rápidas e se confundir, enquanto podem (ou necessitam) ler mais lentamente para realizar o reconhecimento preciso da palavra escrita.

Como exemplo, ao ler a palavra "galo", a pesquisa ao léxico pode ter como resultado gato e galo. Ao conferir, a criança tem que perceber e resolver que é a palavra com /1/ que corresponde ao grafema da palavra escrita. Crianças mais novas podem ser rápidas neste processo e selecionar a palavra errada, enquanto as mais escolarizadas podem ser mais lentas e mais eficientes. Um tempo maior para recuperar uma palavra determinando a imprecisão na leitura indica que suas representações não estão estáveis.

Desta forma, as características das palavras escritas determinam um maior ou menor tempo de recuperação durante a leitura, de acordo com a fase de desenvolvimento e de escolarização em que a criança se encontra. Crianças mais novas e menos escolarizadas apresentam e necessitam de mais tempo para reconhecer a palavra escrita, em decorrência do início do processo de armazenamento e consolidação das representações do material escrito. Já as mais velhas e escolarizadas, pelo maior tempo de exposição ao material e pelo amadurecimento dos processos cognitivos, já podem ativar palavras mais rapidamente, embora lentifiquem este 
processo quando as características do material são mais complexas ou menos conhecidas. O perfil da leitura em cada série, no que se refere aos tipos de erros, é conseqüência do caminho utilizado para o reconhecimento da palavra escrita e do tempo despendido neste processo.

\section{CONCLUSÕES}

A literatura internacional aponta para a influência da fre- qüência no tempo de latência e na precisão da leitura. Em crianças falantes do português do Brasil, verificou-se o efeito de frequiência na leitura, que, neste estudo, variou de acordo com as características do material escrito (extensão, regularidade e complexidade silábica) e a escolaridade, produzindo diferentes tempos de latência em cada tipo de palavra e série. A interação do tempo de latência com as características do material e a escolaridade indicam a relação entre estes fatores durante o desenvolvimento.

\begin{abstract}
Purpose: To verify the influence of frequency of written words and schooling on latency time for reading. Methods: Seventy-four children of both genders from $2^{\text {nd }}, 3^{\text {rd }}$ and $4^{\text {th }}$ grades of public school $(\mathrm{m}=9.6 \mathrm{y})$, without developmental alterations or scholar difficulties complaints took part of this study. It was carried out a loud voice reading test of isolated words with a program especially created for this study. These words were characterized by low and high frequency, varying in regularity, extension and syllabic complexity. Latency time was measured in milliseconds. Results: Data showed that latency time was shorter for the reading of frequent words and there was an interaction between grade and type of word. The negative correlation between latency time and number of correct answers was significant only for low frequency words in $3^{\text {rd }}$ and $4^{\text {th }}$ grades. Conclusion: Frequency had different influences on latency time and accuracy of reading, when words characteristics and schooling were taken into account.
\end{abstract}

Keywords: Children; Reading; Reaction time; Software

\title{
REFERÊNCIAS
}

1. Morais J. A arte de ler. São Paulo: UNESP; 1985.

2. Ziegler JC, Goswami U. Reading acquisition, developmental dyslexia, and skilled reading across languages: a psycholinguistic grain size theory. Psychol Bull. 2005;131(1):3-29.

3. Nergard-Nilssen T. Word-decoding deficits in Norwegian: the impact of psycholinguistic marker effects. Read Writ. 2006;19(3):265-90.

4. Pinheiro AMV, Parente MAMP. Estudo de caso de um paciente com dislexia periférica e as implicações dessa condição nos processamentos centrais. Pró-Fono. 1999;11(1):115-23.

5. Coltheart M, Rastle K, Perry C, Langdon R, Ziegler J. DRC: A dual route cascaded model of visual word recognition and reading aloud. Psychol Rev. 2001;108(1):204-56.

6. Coltheart M, Curtis B, Atkins P, Haller M. Models of reading aloud: Dual route and parallel - distributed - processing approaches. Psychol Rev. 1993;100:589-608.

7. Ellis AW. Leitura, escrita e dislexia: uma análise cognitiva. 2a ed. Porto Alegre: Artes Médicas; 1995.

8. Taft M. Reading and the mental lexicon. Hove (U.K.): Lawrence Erlbaum Associates; 1991.

9. Balota DA, Law MB, Zevin JD. The attentional control of lexical processing pathways: reversing the word frequency effect. Mem Cognit. 2000;28(7):1081-9.

10. Welbourne SR, Lambon Ralph MA. Using parallel distributed processing models to simulate phonological dyslexia: the key role of plasticity-related recovery. J Cogn Neurosci. 2007;19(7):1125-39.

11. Lecours AR, Parente MAMP, organizadores. Dislexia: implicações do sistema de escrita do português. Porto Alegre: Artes Médicas; 1997.

12. Ziegler JC, Perry C, Ma-Wyatt A, Ladner D, Schulte-Körne G. Developmental dyslexia in different languages: language-specific or universal? J Exp Chil Psychol. 2003;86(3):169-93.

13. Alija M, Cuetos F. [Effects of the lexical-semantic variables in visual word recognition]. Psicothema. 2006;18(3):485-91. Spanish.

14. McDougall P, Borowsky R, MacKinnon GE, Hymel S. Process dissociation of sight vocabulary and phonetic decoding in reading: a new perspective on surface and phonological dyslexias. Brain Lang. 2005;92(2):185-203.
15. Kessler B, Treiman R, Mullennix J. Phonetic biases in voice key response time measurements. J Mem Lang. 2002;47(1):145-71.

16. Stanovich KE, West RF. On priming by a sentence context. J Exp Psychol Gen.1993;112(1):1-36.

17. Moser DC, Fridriksson J, Healy EW. Sentence comprehension and general working memory. Clin Linguist Phon. 2007;21(2):147-56.

18. Foster KI, Chambers SM. Lexical access and naming time. J Verbal Learn Verbal Behav. 1973;12:627-35.

19. Sprenger-Charolles L, Siegel LS, Béchennec D, Serniclaes W. Development of phonological and orthographic processing in reading aloud, in silent reading, and in spelling: a four-year longitudinal study. J Exp Child Psychol. 2003;84(3):194-217.

20. Holtgraves T, Han TL. A procedure for studying online conversational processing using a chat bot. Behav Res Methods. 2007;39(1):156-63.

21. Barrow IM, Holbert D, Rastatter MP. Effect of color on developmental picture-vocabulary naming of 4-, 6-, and 8-year-old children. Am J Speech Lang Pathol. 2000;9(4):310-8.

22. Santiago J, MacKay DG, Palma A, Rho C. Sequential activation processes in producing words and syllables: Evidence from picture naming. Lang Cogn Process. 2000;15(1):1-44.

23. Bates E, D’Amico S, Jacobsen T, Székely A, Andonova E, Devescovi A, et al. Timed picture naming in seven languages. Psychon Bull Rev. 2003;10(2):344-80.

24. Alario FX, Ferrand L, Laganaro M, New B, Frauenfelder UH, Segui J. Predictors of picture naming speed. Behav Res Methods. 2004;36(1):140-55.

25. Stivanin L, Scheuer CI. Tempo de latência e exatidão para a leitura e nomeação em crianças escolares: estudo piloto. Educ Pesq. 2005;31(3):425-36.

26. Stivanin L, Scheuer CI. Comparação do tempo de latência entre nomeação e leitura em escolares. Psicol Estud. No prelo 2007.

27. Almeida PM, Wickerhauser H. O critério ABA-ABIPEME: em busca de uma solução. Um estudo e uma proposta submetidos à $\mathrm{ABA}-$ Associação Brasileira de Anunciantes e ABIPEME - Associação Brasileira dos Institutos de Pesquisa de Mercado. São Paulo; 1991. 
28. Pinheiro AMV. Contagem de freqüência de ocorrência de palavras expostas a crianças na faixa de pré-escola e séries iniciais. Software produzido pela Associação Brasileira de Dislexia - ABD; 1996.

29. Frith U, Wimmer H, Landerl K. Differences in phonological recoding in German- and English- speaking children. Scient Stud Read. 1998;2(1):31-54.

30. Salles JF, Parente MAMP. Relação entre os processos cognitivos envolvidos na leitura de palavras e as habilidades de consciência fonológica em escolares. Pró-Fono. 2002;14(2):175-86.
31. Caccamise D, Synder L. Theory and pedagogical practices of text comprehension. Top Lang Dis. 2005;25(1):5-20.

32. Goff DA, Pratt C, Ong B. The relations between children's reading comprehension, working memory, language skills, and components of reading decoding in a normal sample. Read Writ. 2005;18(7-9):583616. 